\title{
On the Circulation of a Homogeneous Ocean Induced by the Presence of Continental Slopes
}

\author{
Peter D. Killworth \\ Scripps Institution of Oceanography, University of California-San Diego, La Jolla 92037 \\ (Manuscript received 26 April 1972, in revised form 30 June 1972)
}

\begin{abstract}
ABSTRAC'T
The three-dimensional circulation of a steady, frictional, homogeneous ocean forced by a wind stress varying sinusoidally in the northward direction is examined, with reference to the effects of continental slopes. It is found that the local circulation pattern is considerably altered by the presence of slopes, although the global pattern is not. Moderately strong eastern boundary currents, of order $1-3 \mathrm{~cm} \mathrm{sec}^{-1}$, are predicted, flowing in the same direction as those observed. Areas of secondary up-or downwelling are found near the bottom of the continental slope, even in the absence of a local long-shore wind stress, again agreeing qualitatively with observations.

The northward, intense (linear) return flow on the western boundary is again modified. It consists of a wide weak flow over the slope, and a narrower strong flow over the flat bottom; both flows have a northward flux of the same order, however.
\end{abstract}

\section{Introduction}

The early theories of large-scale ocean circulation (Sverdrup, 1947; Stommel, 1948; Munk, 1950) were very successful in predicting the gross features of ocean flow. The formulations all employed a transport streamfunction representing the vertically integrated flow. Stommel (1955) pointed out that such a formulation concealed valuable information about the $d y-$ namics. More recently, linear, three-dimensional circulation theories of Pedlosky $(1968,1969)$ and Johnson (1968) have been successful in analyzing ocean flow in regions of other than strong boundary currents, which are believed to be inertially controlled (e.g., Bryan, 1963). These theories were able to cast light on where the surface Ekman flux goes after impinging on a vertical coastline (a problem which is concealed if a transport streamfunction is used). These theories have all involved the ubiquitous eddy coefficients of viscosity, which will also be used here.

The models are unanimous in claiming that the surface Ekman flux is turned in a thin downwelling side layer, and is redistributed into the interior of the fluid. These effects have been studied by Tomczak (1970) and Janowitz (1970). Observations off the west coast of Africa (Hart and Curie, 1960; Jones and Folkard, 1970) and over Rockall Bank (Ellett, 1968) clearly show a corresponding up- or downwelling. However, the observations off Africa also show an area of secondary upwelling some way from the coast, which the above theories do not predict.

Most of the above models also possess bottom frictional layers. Such layers, however, are relatively un- important to the dynamics. Occasionally they interact with the flow above them [e.g., Pedlosky (1968) finds a layer on the northern boundary of an ocean in which the bottom layer helps to complete the circulation], but over most of the ocean the bottom layer is purely passive and contains only little flux (Johnson et al., 1971). However, it is known that homogeneous models "feel" bottom topography rather more than observations would suggest (e.g., Holland, 1967; Clarke and Fofonoff, 1969).

We shall consider here the physically more realistic case of an ocean with boundaries possessing continental slopes. In this case, the surface flux, after impinging on the coast, must return down the continental slope in a layer which combines the properties of a vertical side-wall and a horizontal bottom layer. This combination produces a bottom stress which increases with slope, and is sufficiently strong to affect the flow over the slope to a considerable extent. ${ }^{1}$

Some of the predictions of the model to be described include eastern boundary currents of order $1-3 \mathrm{~cm} \mathrm{sec}^{-1}$, an area of secondary upwelling at the bottom of the continental slope produced by shear in the horizontal velocities, and upwelling in regions without local wind stress, driven by pumping from other areas of the coast. In addition, we shall examine the linear western boundary layer in the presence of a continental slope.

\footnotetext{
${ }^{1}$ Such a change (although with differing dynamics) is, of course, not confined to steady theories [see, for example, Rhines $(1970)]$.
} 


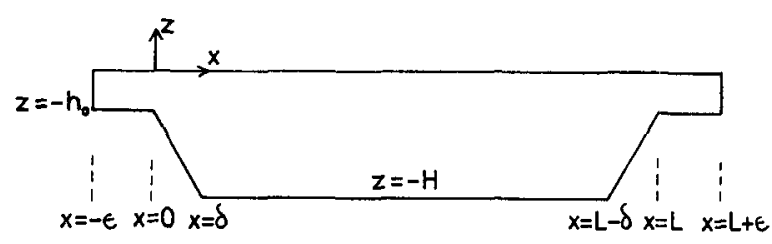

FIG. 1. Cross section of ocean basin: $x$ east, $z$ up, $y$ north.

\section{The model}

We consider a basin whose shape in the plane $y=$ constant is given in Fig. 1 , where $L$ is a planetary length scale for axes $x$ (east) and $y$ (north); the vertical axis is denoted by $z$. The regions $x<0, x>L$ represent the continental shelves on the west and east coasts of the basin; the regions $0<x<\delta, L-\delta<x<L$ the continental slopes. The basin is considered formally to be without boundaries on latitudinal circles, and lies on a $\beta$ plane [i.e., the Coriolis vector $\left.f(y)=f_{0}+\beta y\right]$ in the Northern Hemisphere $\left(f_{0}>0\right)$.

We assume the following: the fluid is homogeneous, incompressible, and hydrostatic, with density $\rho_{0}$; the motion is steady; the velocities are sufficiently small for nonlinear effects to be negligible; constant edd $y$ viscosities $\nu_{I I}, \nu_{V}$ corresponding to lateral and vertical mixing are employed; variations in the $y$ direction on scales smaller than $L$ are neglected; and the dynamics within the thin vertical boundary layers of thickness $\left(\nu_{H} / f\right)^{\frac{x}{2}}$ found, for example, by Pedlosky (1968) are neglected. We shall call these layers "direct lateral friction" layers, to distinguish them from layers produced by interaction between viscous shear and bottom frictional effects. The function of these layers is to smooth out whatever discontinuities are formed by boundary layer matching; we shall assume the existence of a well-behaved solution within them. (It will appear a posteriori that the role of these layers is essentially passive.) Thus, in the region we shall call the "interior," i.e., that part of the ocean away from frictional Ekman layers on the surface and the bottom, and outside direct lateral friction layers, the equations of motion are

$$
\begin{aligned}
-f v & =-p_{x}, \\
f u & =-p_{y}+\nu_{H} v_{x x}, \\
p z & =0, \\
u_{x}+v_{y}+w_{z} & =0,
\end{aligned}
$$

where the velocity $\mathbf{u}=(u, v, w)$, and $p=$ pressure $+g \rho_{0} z$, where $g$ is the acceleration due to gravity.

The eastward equation of motion is geostrophic since the region of interest excludes the direct lateral friction layers. Then it may easily be seen that

giving

$$
u_{z}=v_{z}=w_{z z}=0 \text {, }
$$

$$
w_{z}=\frac{1}{h}\left(w_{\mathrm{E}}-w_{\mathrm{B}}\right)
$$

where $w_{\mathrm{E}}$ is the surface Ekman suction given by the vertical component of curl $(\tau / f)$. This corresponds to a surface flux of $\tau^{(y)} / f$ eastward, $\tau^{(x)} / f$ southward. ${ }^{2}$ $w_{B}$ is the bottom Ekman suction: its value, derived in the Appendix, is

$$
w_{\mathrm{B}}=-u h_{x}+\left(\frac{\nu_{H} s^{2}+v_{V}}{2 f}\right)^{\frac{1}{2}}\left[\left(u_{x}+v_{y}\right)+\left(v_{x}-u_{y}\right)\right],
$$

where $s$ is the local gradient $\left|h_{x}\right|$.

Eqs. (1)-(4) can be combined with (7) to yield the vorticity equation

$$
\begin{aligned}
\frac{h}{f^{2}}\left(\nu_{H} p_{x x x x}-\beta p_{x}\right)+w_{\mathbf{E}}-h_{x} & \left(\frac{p_{y}}{f}-\nu_{H-}^{p_{x x x}} f^{2}\right. \\
- & {\left[p_{x}\left(\frac{\nu_{H} s^{2}+\nu_{V}}{2 f^{3}}\right)^{\frac{1}{2}}\right]_{x}=0, }
\end{aligned}
$$

where we have assumed that (where $w_{\mathrm{B}}$ is important) $\partial / \partial x \gg \partial / \partial y,|v| \gg|u|$. Over a flat bottom, (8) becomes

$$
\frac{h}{f^{2}}\left(\nu_{H} p_{x x x x x}-\beta p_{x}\right)+w_{\mathrm{E}}-p_{x x}\left(\frac{\nu_{V}}{2 f^{3}}\right)^{\frac{1}{2}}=0 .
$$

We shall now make two final simplifying assumptions, namely that $f$ is now taken to be constant and equal to $f_{0}$, and that $\partial / \partial y$ is replaced by $i \alpha$ in (8) or (8a). Both these assumptions have been discussed by Schulman and Niiler (1970).

Before non-dimensionalizing, it is worth estimating the effects of various terms. Above a flat bottom, (8a) holds. Two boundary layer thicknesses are possible, $\left(\nu_{H} / \beta\right)^{\frac{2}{3}}$ [Munk, 1950], a balance between the bracketed terms in (8a) and $\left(\nu_{V} f\right)^{\frac{1}{1}} / \beta h$ [Stommel, 1948], a balance between the $\beta$ and the $p_{x x}$ terms. With $f=10^{-4} \mathrm{sec}^{-1}$, $\beta=10^{-13} \mathrm{~cm}^{-1} \mathrm{sec}^{-1} ;\left(\nu_{H} / \beta\right)^{1 / 3}=20,100 \mathrm{~km}$ according as $\nu_{H}=10^{6}, 10^{8} \mathrm{~cm}^{2} \mathrm{sec}^{-1}$. Over a deep ocean $h \approx 4 \times 10^{5}$ $\mathrm{cm}$, and

$$
\frac{\left(\nu_{V} f\right)^{\frac{2}{2}}}{\beta h}=2.5,25 \mathrm{~km} \text { as } \nu_{V}=1,10^{2} \mathrm{~cm}^{2} \mathrm{sec}^{-1},
$$

the Munk layer will dominate the Stommel layer. On the shelf, $h=10^{4} \mathrm{~cm}$, and

$$
\frac{\left(\nu_{V} f\right)^{\frac{1}{2}}}{\beta h}=10^{2}, 10^{3} \mathrm{~km} \text { as } \nu_{V}=1,10^{2} \mathrm{~cm}^{2} \mathrm{sec}^{-1},
$$

and the bottom frictional layer will dominate.

On the slope, the terms in $s \equiv\left|h_{x}\right|$ appear. Assuming the width of the continental slope to be of order 150 $\mathrm{km}, s$ is $3 \times 10^{-2}$. With motions of length scale $150 \mathrm{~km}$, the motion on the slope is geostrophic and the predominant

\footnotetext{
${ }^{2} \tau=\left[\tau^{(x)}, \tau^{(y)}, 0\right]$ is the wind stress.
} 
balance in (8) is (noting that $\nu_{H} s^{2} \gg \nu_{V}$ )

$$
\left[\frac{-\beta h}{f^{2}} p_{x}\right]+w_{\mathrm{E}}-\frac{i \alpha h_{x} p}{f}-p_{x x} s\left(\frac{\nu_{I I}}{2 f^{3}}\right)^{\frac{1}{2}}=0
$$

where $\alpha$ is of order $10^{-8} \mathrm{~cm}^{-1}$, and the bracketed term may be important toward the bottom of the slope. We note that the bottom Ekman layer is controlled by horizontal rather than vertical viscosity, for all reasonable values of $\nu_{I I}, \nu_{V}$, and realistic bottom gradients. This does not mean, however, that the continental slopes act as "vertical walls" for the flow in the rest of the basin.

We non-dimensionalize as follows:

$$
\left.\begin{array}{l}
(x, y)=L\left(x^{\prime}, y^{\prime}\right) ; z=H z^{\prime} ;\left(u, v, \frac{L w}{H}\right)=V^{*}\left(u^{\prime}, v^{\prime}, w^{\prime}\right) \\
\tau=\tau_{0} \tau^{\prime} ; p=f_{0} V^{*} L p^{\prime} ; f=f_{0} f^{\prime}=f_{0}\left(1+\beta^{\prime} y^{\prime}\right) ; \alpha=L^{-\prime} \alpha^{\prime}
\end{array}\right\},
$$

and define the Ekman numbers and velocity scale by

$$
\mathrm{E}_{H}=\frac{\nu_{H}}{f_{0} L^{2}}, \quad \mathrm{E}_{V}=\frac{\nu_{V}}{f_{0} H^{2}} \quad V^{*}=\frac{\tau_{0}}{\left(f_{0} \nu_{V}\right)^{\frac{1}{2}}} .
$$

Then dropping dashes, (1) and (2) become

$$
\begin{aligned}
& v=p_{x} \\
& u=-i \alpha p+\mathbf{E} p_{x x x},
\end{aligned}
$$

while (3) and (4) retain their original form. We shall assume for convenience that $\mathrm{E}_{H}=\mathrm{E}_{V} \equiv \mathrm{E}$. This has the effect of re-scaling the wind forcing term, which merely rescales the entire problem (because of linearity). We shall also use the same notation $\delta, h_{0}$ for the non-dimensional quantities $\delta / L, h_{0} / H$. Eq. (8) becomes

$$
\begin{aligned}
h\left(\mathrm{E}_{x x x x}-\beta p_{x}\right)-i \alpha h_{x} p+\mathrm{E} h_{x} p_{x x x}+w_{\mathrm{E}} & \\
& -\frac{\mathrm{E}^{\frac{1}{2}}}{2^{\frac{1}{2}}} p_{x x}|\tan K|=0,
\end{aligned}
$$

where $\tan K$, the nondimensional $h_{x}$, is $1 / \delta$, which will be considered a large number. (Had we retained only the horizontal viscosity in the bottom Ekman layer, $\sec K$ would have replaced $\tan K$. For small $\delta$ these are identical.)

The three small quantities in the problem, with $H=4$ $\times 10^{5}, L=6 \times 10^{8} \mathrm{~cm}$, are: $h_{0}=\frac{1}{4} \times 10^{4} \mathrm{~cm} / 10^{5} \mathrm{~cm}=2.5$ $\times 10^{-2} ; \delta=150 \mathrm{~km} / 6000 \mathrm{~km}=2.5 \times 10^{-2} ; \mathrm{E}\left(=\mathrm{E}_{H}\right)=2.8$ $\times\left(10^{-8}, 10^{-6}\right)$ as $\nu_{H}=\left(10^{6}, 10^{8}\right) \mathrm{cm}^{2} \mathrm{sec}^{-1}$. We note for future use that the relation between these parameters is

$$
\delta \approx h_{0} \approx \mathrm{E}^{\frac{1}{2}} \ll 1,
$$

and that the vertical ${ }^{3}$ extent, non-dimensionally, of the bottom Ekman layer on the slope is $\mathrm{E}^{\frac{1}{2}} \sec K \approx \mathrm{E}^{\frac{1}{2}} / \delta \approx h_{0}$. This means that the surface and bottom Ekman

\footnotetext{
${ }^{3}$ The horizontal extent is $\mathrm{E}^{\mathrm{l}}$ and therefore much smaller than $\delta$.
}

layers "overlap" at the top of the slope. Within this overlap region, horizontal and vertical friction are both important, and the problem becomes intractable analytically. As a result of this, seaward boundary conditions becomes unavailable for the solution of the problem on the shelf. However, as we shall see in the next section, motions on the shelf do not affect motions elsewhere, making a more detailed study unnecessary.

Lastly, the Ekman suction at the surface, under the assumptions of sinusoidal $y$ variation, is

$$
w_{\mathrm{E}}=O\left(\mathrm{E}^{\frac{1}{2}}\right)=\mathrm{E}^{\frac{1}{2}}\left[\tau_{x}{ }^{(y)}+(\beta-i \alpha) \tau^{(x)}\right] .
$$

\section{The solutions on the eastern slope and interior}

The whole of the slope region, width $\mathrm{O}\left(\mathrm{E}^{\frac{1}{4}}\right)$, is technically a boundary layer. It is fairly unusual, however, in that the motion within it is completely geostrophic. The equation satisfied on the eastern slope, away from thinner boundary layers, is

$$
-\beta h p_{x}-i \alpha h_{x} p-\frac{\mathbf{E}^{\frac{1}{2}}}{2^{\frac{1}{2}} \cos K} p_{x x}=-w_{\mathrm{E}}
$$

Schulman and Niiler (1970) used this equation without the frictional term, and found oscillations in the sign of the streamfunction over regions of changing depth, caused by the omission, as here, of north-south boundaries, and the partial tendency of the solution to conserve potential vorticity. Here, however, (10) reduces to

$$
\mathbf{u} \cdot \nabla(\beta y-\log h)+\frac{\mathrm{E}^{\frac{1}{2}}}{2^{\frac{1}{2}}} \frac{\left|h_{x}\right| \zeta}{h}=w_{\mathrm{E}},
$$

where $\zeta \approx v_{x}$, the relative vorticity, and for zero $w_{\mathrm{E}}$ we see that the "linearized" potential vorticity, $\beta y-\log h$, may be altered on a streamline by vortex stretching due to suction by the bottom Ekman layer, if $\left|h_{x}\right|$ is large enough (conversely on gentle slopes, the results of Schulman and Niiler will apply). Putting $\xi=1-x,(10)$ becomes

$$
\frac{\mathrm{E}^{\frac{1}{2}}}{2^{\frac{1}{2}}} p_{\xi \xi}-\beta \xi p_{\xi}-i \alpha p=\delta w_{\mathrm{E}}
$$

with solution

$$
p=\frac{i \delta w_{\mathrm{E}}}{\alpha}+A \bar{M}(\xi)+B \bar{U}(\xi),
$$

where

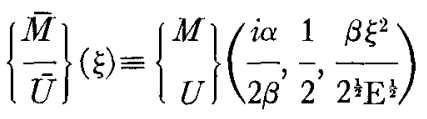

and

$$
\left\{\begin{array}{c}
M \\
U
\end{array}\right\}(a, b, z)
$$

are the confluent hypergeometric functions (Abramo- 
witz and Stegun, 1965. To determine $A$ and $B$ we must apply boundary conditions. At the top of the slope, the lateral-bottom frictional layer of thickness $\left(\delta h_{0}\right)^{\frac{1}{1}} \mathrm{E}^{\frac{1}{4}}$ has been submerged within the direct lateral friction layer, and so we may only apply one boundary condition, namely that of mass continuity. The surface Ekman flux in the eastward direction, $\mathrm{E}^{\frac{1}{2}} \tau^{(y)}(1)$, flows toward the top of the eastern slope. At this point it may do three things, consistent with mass conservation:

(i) it may flow onto the shelf and turn northward (or southward)

(ii) it may turn and flow westward in the interior of the fluid

(iii) it may turn and flow down the slope in the bottom Ekman layer; or any combination of these.

Case (i) can be shown to be unphysical. The incoming surface flux abutting into the shelf region is dimensionally $\tau^{(y)} / f \rho_{0}$, of order $10^{4} \mathrm{~cm}^{2} \mathrm{sec}^{-1}$ for $\tau^{(y)} \approx 1 \mathrm{dyn} \mathrm{cm}{ }^{-2}$, $\rho_{0}=1 \mathrm{gm} \mathrm{cm}^{-3}, f \approx 10^{-4} \mathrm{sec}^{-1}$. If the shelf region, assumed to be $10^{4} \mathrm{~cm}$ deep, could accept this, continuity would imply that the northward velocity change by $1 \mathrm{~cm} \mathrm{sec}^{-1}$ per distance $\mathrm{E}$ along the coast, where $\mathrm{E}$ is the shelf width. For $\mathrm{E} \approx 10 \mathrm{~km}$, velocities as large as $50 \mathrm{~cm} \mathrm{sec}^{-1}$ would be produced along a $500-\mathrm{km}$ coastline, and these are much higher than observed, and hence unacceptable. We may also show that case (ii) cannot occur. The eastward flux in the interior is $u h$. Because of the "overlap" of the top and bottom Ekman layers, the depth of the interior of the fluid is zero on $x=1$. To carry a finite interior flux as $h \rightarrow 0$ would mean $u \rightarrow \infty$, which is not allowed by the solutions (11), both of which are well-behaved at $x=1$. We are left, therefore, only with the possibility that the flux is returned, at least in the neighborhood of $x=1$, by the bottom Ekman layer.

To understand the difference between this situation and the Pedlosky (1968) $\mathrm{E}^{\frac{1}{2}}$ side-wall layer, it will be necessary to examine the latter. Assume $\tau^{(y)}=1$, corresponding to downwelling at the coast. Fig. 2a shows schematically the fluxes (represented by a double arrow) and the velocities (by a single arrow) in a projection onto the $x, z$ plane, of the Pedlosky theory. The
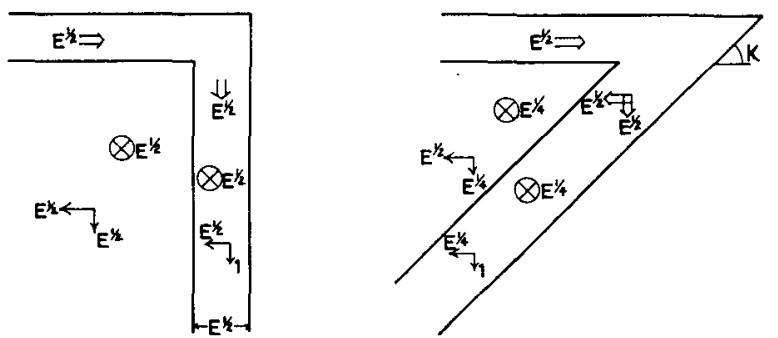

FIG. 2. Velocities and fluxes in vertical (a) and sloping (b) wall cases. Single arrows represent velocities in the plane, circles with crosses velocities normal to plane; and double arrows represent fluxes in plane.
$O\left(E^{\frac{1}{2}}\right)$ surface flux flows down in the direct lateral friction layer, thickness $\mathrm{O}\left(\mathrm{E}^{\mathrm{l}}\right)$, forcing an $\mathrm{O}(1)$ vertical velocity. This, by mass conservation, forces a westward velocity $\mathrm{O}\left(\mathrm{E}^{\frac{1}{2}}\right)$ which, when integrated from $z=-1$ to 0 , removes this flux back into the interior.

Fig. 2b, on the other hand, shows the situation studied here. The $\mathrm{O}\left(\mathrm{E}^{\frac{1}{2}}\right)$ surface flux is now returning in the bottom layer. Mass conservation implies that the vertical flux in the bottom layer (near $x=1$ ) is of order $\mathrm{E}^{\frac{1}{2}}$. The horizontal width of the bottom layer is $\mathrm{E}^{\frac{1}{3}} \csc K$, i.e., $O\left(E^{\frac{1}{2}}\right)$, so the vertical velocities remain $O(1)$ as in the standard theory, but with differing values. Mass conservation also implies a horizontal flux of order $\mathrm{E}^{\frac{1}{2}}$ within the bottom layer. The vertical width of the bottom layer is $\mathrm{E}^{\frac{2}{2}} \sec K \approx \mathrm{E}: \frac{1}{4}$ so horizontal velocities of order $\mathrm{E}^{\frac{1}{4}}$ are required. Our boundary layer solution shows that these must be associated with velocities in the interior, also $O\left(E^{\frac{1}{2}}\right)$, which can only be northward by continuity [for eastward velocities $\mathrm{O}\left(\mathrm{E}^{\frac{1}{4}}\right)$ would be associated, by continuity, with northward velocities $\mathrm{O}(1)]$. We may note that this description shows clearly the fundamental difference in formulation between the lateral-vertical friction model and the more usual bottom stress model. In the latter, the bottom stress is a function (usually quadratic) of the interior velocity alone, and is independent of bottom slope. Direct measurements of bottom stress on slopes near Bermuda suggest that the bottom stress evaluated by the quadratic drag law is probably more accurate than that calculated from the frictional model presented here (Niiler, private communication).

The northward interior velocities, $\mathrm{O}\left(\mathrm{E}^{1}\right)$, act over an area $\mathrm{O}(1)$ (in $z) \times O\left(\mathbf{E}^{\frac{1}{}}\right)$ (in $\left.x\right)=O\left(\mathrm{E}^{\frac{1}{t}}\right)$, giving a northward flux on an eastern boundary of order $E^{\frac{1}{2}}$, of the same order as the flux in the western Munk layer in the Pedlosky theory. We shall see later, however, that while the flux is formally of the same order, actual computation reveals it to be small numerically. Note that this mechanism produces longshore velocities with the same sign as the local northward wind stress (in the Northern Hemisphere). It is quite distinct from the relative, baroclinic, coastal current predicted by Sverdrup et al. (1942) as a result of up- or downwelling, although it acts in the same sense. Mass continuity on $x=1$ is then the condition on the interior $v$ that the flux in the bottom Ekman layer be minus the surface flux, or

i.e.,

$$
p_{x}=\delta 2^{\frac{1}{2}} \tau^{(y)}(1) \text { on } x=1,
$$

$$
A \bar{M}^{\prime}(0)+B \bar{U}^{\prime}(0)=-\delta 2^{\frac{1}{2}} \tau^{(y)}(1)
$$

where primes denote differentiation. The properties of $\bar{M}$ and $\bar{U}$ are such that $\bar{M}^{\prime}(0)=0$, and so

$$
B=\frac{-\delta 2^{\frac{1}{2}} \tau^{(y)}(1)}{\overline{U^{\prime}(0)}}=\frac{-i \delta \mathrm{E}^{\frac{1}{2}} 2^{\frac{3}{2}} \Gamma\left(\frac{i \alpha}{2 \beta}+1\right) \beta^{\frac{1}{2}}}{\alpha \Gamma\left(\frac{1}{2}\right)} \tau^{(y)}(1),
$$


which is $\mathrm{O}\left(\mathrm{E}^{3}\right)$. In other words, there are two driving components for the interior velocity field. The first is the surface Ekman suction, which is unimportant here and gives a pressure $\mathrm{O}\left(\mathrm{E}^{\frac{3}{2}}\right)$. The second is the local flux into the coast, giving the dominant contribution to the pressure $\mathrm{O}\left(\mathrm{E}^{\frac{1}{2}}\right)$.

To complete the solution on the slope we must determine $A$. This is found by matching the solution at the bottom of the slope with that for the flat bottom. The general solution of (9) over a flat bottom is of the form

$p=\frac{1}{\beta} \int_{1-\delta}^{x} w_{\mathbf{E}} d x+G$

ta term representing the Munk-layer solution,

where $G$ is an arbitrary constant (note that in Pedlosky's work this was an arbitrary function of $y$; here we have specialized to sinusoidal variations in $y$ ). The equation satisfied in the Munk layer is

$$
\mathrm{E} p_{x x x x}-\beta p_{x}=0 \text {, }
$$

which possesses two oscillatory solutions, damped in the eastward direction, and one solution damped in the westward direction. On the eastern boundary only the latter may be chosen, so that over the flat bottom

$p=\frac{1}{\beta} \int_{1-\delta}^{x} w_{\mathrm{E}} d x+G+F \exp \left\{[x-(1-\delta)] \beta^{1} \mathrm{E}^{-1}\right\}$.

Over the slope, in the neighborhood of $x=1-\delta$, there is a lateral bottom frictional layer in which

$$
\mathrm{E} p_{x x x x}-\frac{\mathrm{E}^{\frac{1}{2}}}{\delta 2^{\frac{1}{2}}} p_{x x}=0 .
$$

Its thickness in $\delta^{\frac{1}{2}} \mathrm{E}^{\frac{1}{2}}$. (The value of $\delta^{\frac{1}{2}} \mathrm{E}^{\frac{1}{2}}$, using the parameters in Section 2, lies between 0.1 and 0.3 of the slope width.) The equation possesses solutions decaying in both directions, and we pick the one which decays eastward. Thus the pressure on the slope is given by

$$
\begin{aligned}
p=\frac{i \delta w_{\mathrm{E}}}{\alpha}+A \bar{M}(\xi)+B \bar{U}(\xi) & \\
& +D \exp \left\{\frac{-[x-(1-\delta)]}{2^{\frac{1}{1}} \delta^{\frac{1}{2}} \mathrm{E}^{1}}\right\},
\end{aligned}
$$

and (14), (15) must be matched at $x=1-\delta$. Examination of (1), (2) yields

$$
[p]_{ \pm}^{+}=\left[p_{x}\right]_{ \pm}^{+}=\left[p_{x x}\right]_{ \pm}^{+}=0,
$$

i.e., $v$ and $v_{x}$ are continuous (no jump in shear). The extra condition comes from integrating (9) across a jump in bottom slope, and gives

$$
\left[p_{x x x}\right] \pm=\frac{\mathrm{E}^{-\frac{x}{2}} p_{x}}{h 2^{\frac{1}{2}}}[\sec K] \pm
$$

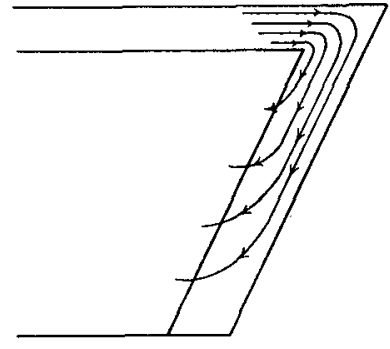

Fig. 3. Pattern of flow into and out of slope layer (downwelling case).

which is directly equivalent to the condition that there is no jump in the total normal flux (i.e., interior and bottom layer fluxes) impinging on $x=1-\delta$. To write down the resulting matching equations we shall use the notation

$$
\lambda=\frac{\beta \delta^{2}}{2^{\frac{1}{2}} E^{\frac{1}{2}}} ;\left\{\begin{array}{c}
M \\
U
\end{array}\right\}(x) \equiv\left\{\begin{array}{c}
M \\
U
\end{array}\right\}\left(\begin{array}{c}
i \alpha \\
2 \beta
\end{array}, \frac{1}{2}, x\right) .
$$

Then, matching $p$,

$$
A M(\lambda)+B U(\lambda)-G=F-D-\frac{i \delta w_{\mathrm{E}}}{\alpha}
$$

(the terms on the left form the principal balance when there is a local surface flux into the coast).

Now, $\bar{M}(\delta)=\left(2^{\frac{1}{3}} \beta \delta / E^{\frac{1}{2}}\right) M^{\prime}(\lambda)$, so, matching $p_{x}$,

$$
\frac{-2^{\frac{1}{2}} \beta \delta}{\mathrm{E}^{\frac{1}{2}}}\left[A M^{\prime}(\lambda)+B U^{\prime}(\lambda)\right]=-\frac{W_{\mathrm{E}}(1)}{\beta}+\frac{\beta^{\frac{1}{3}}}{\mathrm{E}^{\frac{1}{3}}} F+\frac{D}{2^{\frac{1}{2}} \delta^{\frac{1}{2}} \mathrm{E}^{\frac{1}{3}}}
$$

where we have written $w_{\mathbf{E}}(1) \approx w_{\mathbf{E}}(1-\delta)$. Similarly, matching $p_{x x}$,

$$
\begin{array}{r}
\frac{2 \beta^{2} \delta^{2}}{\mathrm{E}}\left[A M^{\prime \prime}(\lambda)+B U^{\prime \prime}(\lambda)\right]+\frac{D}{2^{\frac{1}{2}} \delta \mathrm{E}^{\frac{1}{2}}} \\
=\frac{w_{\mathrm{Ex}}(1)}{\beta}+\frac{\beta^{\frac{2}{5}} F}{\mathrm{E}^{\frac{2}{3}}}-\frac{2^{\frac{1}{\beta}} \beta}{\mathrm{E}^{\frac{1}{2}}}\left[A M^{\prime}(\lambda)+B U^{\prime}(\lambda)\right],
\end{array}
$$

and condition (16b) gives

$$
\frac{\beta}{\mathrm{E}}\left[A M^{\prime}(\lambda)+B U^{\prime}(\lambda)\right]=\frac{\beta}{\mathrm{E}} F-\frac{w_{\mathrm{E}}(1)}{2^{\frac{1}{2}} \beta \mathrm{E}^{\frac{1}{3}}},
$$

the terms in $D$ having vanished identically.

We may solve (17) formally in an asymptotic sense. However, there are two sets of solutions, those terms proportional to the local wind forcing, $\tau^{(y)}(1)$, and those proportional to $w_{E}(1)$. The former terms have the following orders of magnitude:

$A, B, D, G$, order $\mathrm{E}^{\frac{1}{3}}, \mathrm{E}^{\frac{1}{2}}, \mathrm{E}^{\frac{3}{2}}, \mathrm{E}^{\frac{1}{2}}$, respectively; $F$ is zero. When these terms are substituted into (17), $w_{\mathrm{E}}$ vanishes, and the term in $D$ only survives in (17c). The 
terms proportional to $w_{\mathrm{E}}(1)$, however, are of orders

$$
A, B, D, G, F, \text { order } \mathbf{E}^{5 / 6}, \mathbf{E}^{5 / 6}, \mathrm{E}^{11 / 12}, \mathrm{E}^{3 / 4}, \mathrm{E}^{5 / 6}
$$

and we shall ignore these.

The solutions proportional to $\tau^{(y)}(1)$ are:

$$
\begin{gathered}
B=-\mathrm{E}^{\frac{1}{2}} \gamma \tau^{(y)}(1), \\
A=\frac{U^{\prime}(\lambda)}{M^{\prime}(\lambda)} B, \\
G=A M(\lambda)+B U(\lambda)=\frac{-i \mathrm{E}^{\frac{1}{2}} \tau^{(y)}(1)}{\alpha M\left(\frac{1}{2}-\frac{i \alpha}{2 \beta}, \frac{3}{2},-\lambda\right)}, \\
D=\frac{-2^{\frac{1}{3} \beta^{2} \delta^{3}}}{\mathrm{E}^{\frac{1}{2}}}\left[A M^{\prime \prime}(\lambda)+B U^{\prime \prime}(\lambda)\right],
\end{gathered}
$$

where

$$
\gamma=O(1)=\frac{i \delta 2^{\frac{3}{4}} \Gamma\left(\frac{i \alpha}{2 \beta}+1\right) \beta^{\frac{1}{3}}}{\mathrm{E}^{\frac{1}{2}} \alpha \Gamma\left(\frac{1}{2}\right)} .
$$

We note that (18b) implies that $p_{x}=0$ at the bottom of the slope, i.e., $v=0$ there. This in turn implies that all the fluid in the bottom layer has leaked into the interior before the bottom of the slope is reached, giving a qualitative picture as in Fig. 3. This, of course, presents the same aspect to the rest of the ocean as the Pedlosky vertical-wall picture. Then finally we have, on the slope,

$$
\left.\begin{array}{l}
u=-i \alpha\left[A M\left(\frac{i \alpha}{2 \beta}, \frac{1}{2}, \frac{\beta \xi^{2}}{2^{\frac{1}{2}} \mathrm{E}^{\frac{1}{2}}}\right)+B U\left(\frac{i \alpha}{2 \beta^{2}}, \frac{1}{2}, \frac{\beta \xi^{2}}{2^{\frac{1}{2}} \mathrm{E}^{\frac{1}{2}}}\right)\right] \\
v^{\prime}=\frac{-2^{\frac{1}{2}} \beta \xi}{\mathrm{E}^{\frac{1}{2}}}\left[A M^{\prime}\left(\frac{i \alpha}{2 \beta}, \frac{1}{2}, \frac{\beta \xi^{2}}{2^{\frac{1}{2}} \mathrm{E}^{\frac{1}{2}}}\right)+B U^{\prime}\left(\frac{i \alpha}{2 \beta}, \frac{1}{2}, \frac{\beta \xi^{2}}{2^{\frac{1}{2}} \mathrm{E}^{\frac{1}{2}}}\right)\right] .
\end{array}\right\}
$$

\section{Properties of the horizontal velocity field over the eastern slope}

The formal solution (11) and (18) for the pressure field can only be made intelligible by machine calculation of the quantities involved. In all the nondimensional solutions to be presented graphically, $\alpha$ (the wavenumber) and $\beta$ were taken as $\pi$ and $\frac{1}{2}$, respectively. As has been commented already, the $\beta$ term in (8) is important only near the bottom of the slope, or when $\delta \mathrm{E}^{-\frac{1}{2}}>1$. In order to help present the solutions, two new parameters are defined:

$\sigma \equiv \delta / \mathrm{E}^{\frac{1}{4}}, \quad$ a measure of the width of the slope compared to the frictional thickness; $\sigma$ will vary from 0.4 to 2 in the results presented.

$\eta \equiv \xi / \delta, \quad$ a stretched variable which runs from 0 at the top of the slope to 1 at the bottom.
Fig. 4 shows the real and imaginary parts of $v /\left[E^{i} \tau^{(y)}(1)\right]$, and the same for $u /\left[E^{i} \tau^{(y)}(1)\right]$, for $\sigma=0.5,1,2$.

Assume $\tau^{(y)}(1)=1$, corresponding to a local Ekman flux into the coast. Then, on $y=0$, where the stress is at its maximum, the velocities are given by

$$
\operatorname{Re}\left(v_{r}+i v_{i}\right) e^{0}=v_{r},
$$

etc. The velocity is northward over the upper half of the slope, and only when the slope is sufficiently wide $(\sigma>1.7)$ can the velocity be southward over the lower half of the slope at such a value of $y$. Unless $\sigma>1.7$ the $u$ velocity at $y=0$ due to a northward wind stress is westward. One-quarter of a wavelength northward, however, the situation has changed enormously; the velocities are now proportional to

$$
\operatorname{Re}\left(v_{r}+i v_{i}\right) e^{i \pi / 2}=-v_{i},
$$

etc. This means that a much smaller northward velocity exists, but, unlike the case at $y=0$, it cannot change sign for any $\eta$. The $u$ component is eastward near the coast, and westward farther away from it. In other words, at the latitude corresponding to the center of a gyre $\left(y=\frac{1}{2}\right)$ there is always a point on the eastern slope for which $u=0$. [A similar phenomenon, but with different dynamics, was found by Janowitz (1970), who noted the application to the theory of coastal pollution; i.e., that pollutants originating near a coastline are confined to remain near it. For sufficiently wide coastal slopes $(\sigma>1.7)$ such a point exists at every latitude.]

An example of the horizontal circulation within a gyre is given in Fig. 5.

It has been observed that there is a northward flux of order $\mathrm{E}^{\frac{1}{2}}$ on the eastern slope. This flux is

$$
\int_{1-\delta}^{1} h p_{x} d x
$$

and may easily be obtained from (8) by integration, giving

$$
f l u x=\frac{i \alpha}{\beta-i \alpha} G-\frac{E^{\frac{1}{2}} \tau^{(y)}(1)}{\beta-i \alpha}=\frac{E^{\frac{1}{2}} \tau^{(y)}(1)}{\beta-i \alpha}\left[\frac{i \alpha e^{\lambda}}{\beta M^{\prime}(\lambda)}-1\right] .
$$

A graph of the real and imaginary parts of this flux is given in Fig. 6. The real part, i.e., the flux on $y=0$ corresponding to $\tau^{(y)}(1)=1$, reaches a maximum at $\sigma=1.4$. That this should happen is easily seen from Fig. 4. The northward velocity near the bottom of the slope gives a large contribution to the northward flux; for $\sigma \geqslant 1$, this velocity decreases with $\sigma$, finally becoming negative near $\sigma=2$. The imaginary part of $v$, however, corresponding to (minus the northward flux on $y=\frac{1}{2}$ ) does not reach a maximum until $\sigma \approx 2$. The dimensional equivalent of the above gives northward velocities of order $1-3 \mathrm{~cm} \mathrm{sec}^{-1}$ for $\tau \approx 1$ dyn $\mathrm{cm}^{-2}$. This seems a little low compared with measured long-shore currents of 

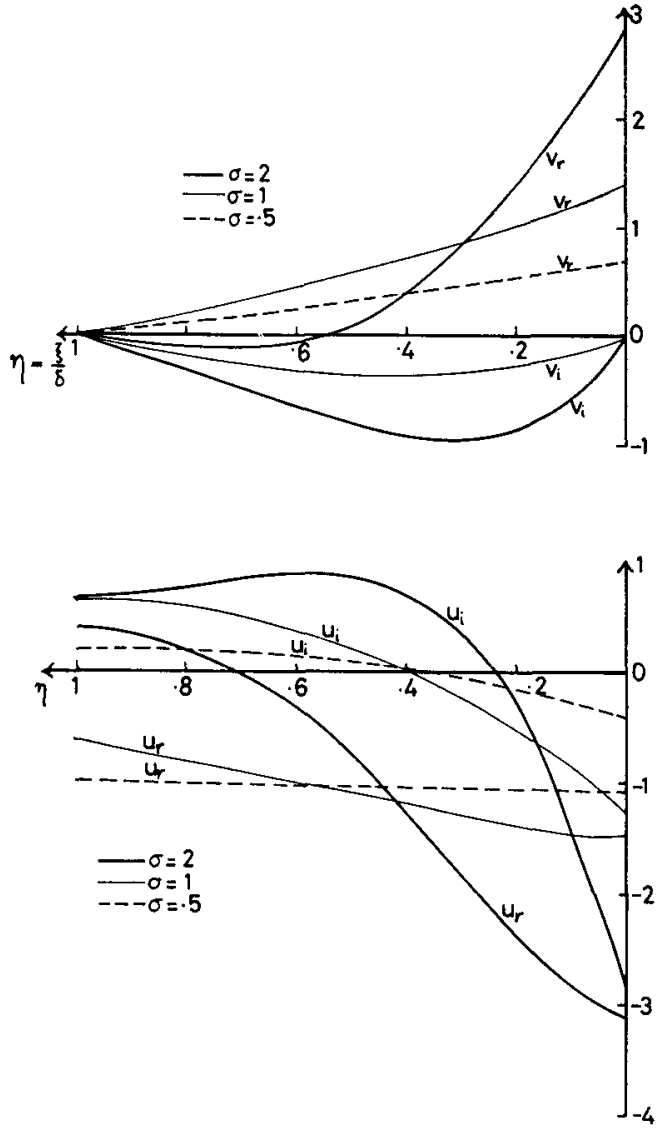

FIG. 4. Velocity components $u, v$ on eastern slope as functions of $\eta \equiv \xi / \delta: v=\mathrm{E}^{\frac{1}{4}} \tau^{(y)}(1)\left[v_{r}+i v_{i}\right] ; u=\mathrm{E}^{\frac{1}{2}} \tau^{(y)}(1)\left[u_{r}+i u_{i}\right]$.

order $5-10 \mathrm{~cm} \mathrm{sec}^{-1}$. The pressure terms (i.e., $A, B$ ) are equivalent to about $1 \mathrm{~cm}$ of water for the same wind stress.

The dimensional equivalent of $(20)$ is

$$
\operatorname{flux}=\frac{\tau^{(y)}(L)}{\beta-i \alpha f}\left\{\frac{1}{M\left[\begin{array}{c}
1 \\
\left.\frac{i \alpha f}{2}-\frac{3}{2 \beta}, \frac{3}{2}, \frac{-\beta \delta^{2}}{\left(2 \nu_{H} f\right)^{\frac{1}{2}}}\right]
\end{array}\right]}-1\right\}
$$

With $f=10^{-4} \sec ^{-1},(21)$ gives a northward flux of at most 1 sverdrup $\left(10^{6} \mathrm{~m}^{3} \mathrm{sec}^{-1}\right)$. This is quite a reasonable value; no strong current systems are observed on east coasts.

We have seen, therefore, that no large fluxes may be achieved on an eastern coast due to this mechanism. Mass conservation implies that a northward, $O\left(E^{\frac{1}{2}}\right)$ flux on $x=1$ must be balanced by a corresponding change in the western boundary layer. Since the eastern flux is of order 1 sverdrup, this mechanism cannot explain the observed flux of the Gulf Stream, which is at least 50 sverdrups and a factor of 2 or 3 larger than that predicted by a linear theory.

This model cannot explain the countercurrent found at the top of the continental slope in the Benguela Cur-

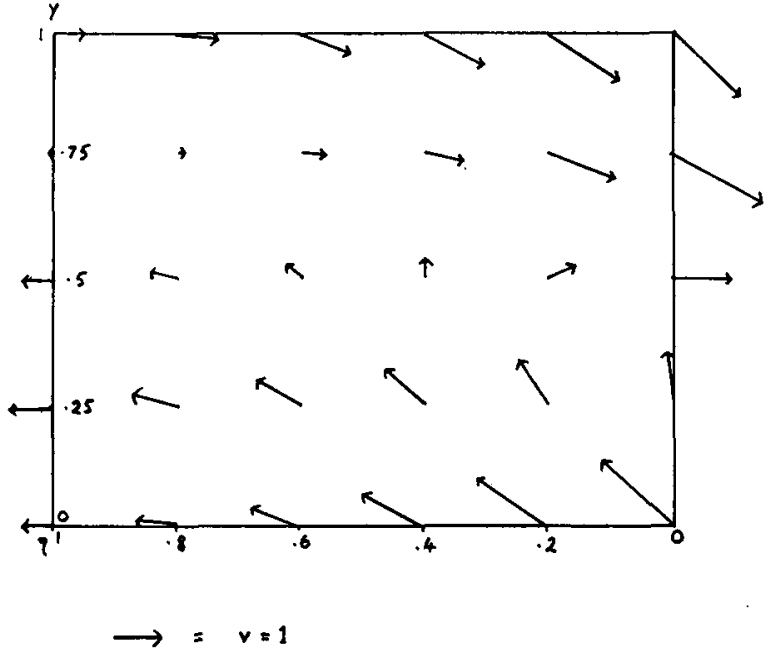

FIG. 5. Horizontal current vectors in the $(\eta, y)$ plane for $\sigma=1$, $\tau^{(y)}=1$, corresponding to surface inflow (i.e., eastward flow) at $y=0$, outflow at $y=1$. The velocity plotted is $\left(\mathrm{E}^{-\frac{1}{2}} u, \mathrm{E}^{-\frac{1}{2}} v\right)$.

rent by Hart and Currie (1960). Neither the frictional, barotropic motion described here, nor the relative baroclinic effects of Sverdrup et al. (1942) predict such a current. It is suggested that Hart and Currie's current is a baroclinic phenomenon. It is probable that the relevant density gradient (light water on the shelf, heavier water on the slope) is caused by the effective isolation of the continental shelf by the return mechanism of Section 3, and upwelling on the slope.

\section{The deep upwelling velocity on the eastern slope}

Away from Ekman layers, the vertical velocity over the slope, within the $\delta^{\frac{1}{2}} \mathrm{E}^{\frac{1}{l}}$ lateral bottom friction layer, and even the $\mathbf{E}^{\frac{1}{2}}$ direct lateral friction layer, is formally always $\mathrm{O}\left(\mathrm{E}^{\mathrm{i}}\right)$. Over the flat bottom it is smaller, $\mathrm{O}\left(\mathrm{E}^{\mathrm{i}}\right)$. However, when we actually calculate the magnitude

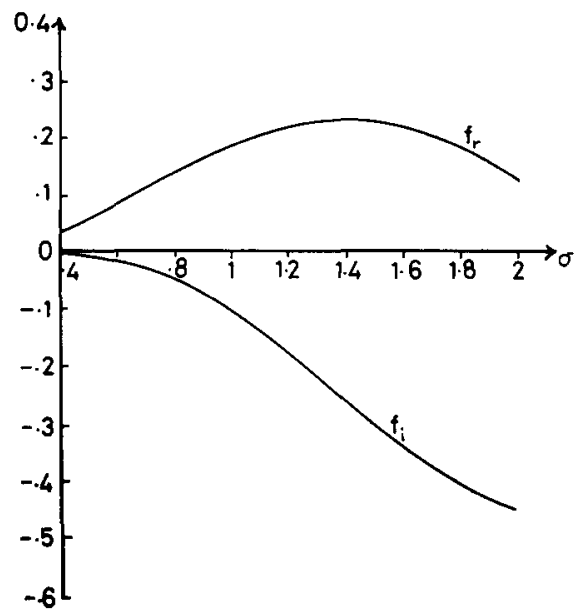

Fig. 6. Northward flux on eastern slope as a function of $\sigma$. Flux $=\mathbf{E}^{\underline{i}} \boldsymbol{\tau}^{(y)}(1)\left[f_{r}+f_{i}\right]$. 
of $w$ we find again differences of order 10 between $w$ over the slope and $w$ within the $\delta^{\frac{1}{2}} \mathrm{E}^{\frac{1}{2}}$ layer.

We have, over the slope,

$$
\begin{gathered}
\text { i.e., } \quad w_{\mathrm{B}}=h\left(u_{x}+v_{y}\right), \quad \text { as } w_{\mathrm{E}}=\mathrm{O}\left(\mathrm{E}^{\frac{1}{2}}\right), \\
w_{\mathrm{B}}=\frac{\beta \eta}{\sigma \mathrm{E}^{\frac{1}{2}}} p_{\eta} .
\end{gathered}
$$

Substituting into the solution (18), we obtain

$$
\begin{aligned}
w_{\mathrm{B}}=\frac{i \pi \beta \eta^{2}}{2^{\frac{1}{2}} \mathrm{E}^{\frac{1}{1}}}\left[2 A M\left(\frac{i \alpha}{2 \beta}+1, \frac{3}{2}, \lambda\right)\right. & \\
& \left.-B U\left(\frac{i \alpha}{2 \beta}+1, \frac{3}{2}, \lambda\right)\right]
\end{aligned}
$$

as a function of $\eta ; w$ itself is $-z w_{\mathrm{B}} h^{-1}$. A graph of the real and imaginary parts of $w_{B} /\left[E^{\frac{1}{2}} \tau^{(y)}(1)\right]$ is given in Fig. $7: w_{\mathrm{B}}$ tends to zero on both $\eta=0$ and $\eta=1$; the former because the depth tends to zero, the latter as $p_{x}=0$ on $\eta=1$. We note that uniformly $|w|<0.2 \mathrm{E}^{\frac{1}{4}}\left|\tau^{(y)}(1)\right|$. This order is of course smaller than the $O(1)$ vertical velocities in the bottom Ekman layer, which occur at the top of the slope. Thus, the vertical velocities given in Fig. 7 would be difficult to detect in any observational data.

If we now turn to the lateral bottom friction layer, near $\xi=\delta$, a different picture emerges: $w_{\mathrm{B}}$ is still given by (23), although $\left|u_{x}\right| \gg\left|v_{y}\right|$ in this layer. The pressure is given as

$$
p=D \exp \left\{-[x-(1-\delta)] / 2^{\frac{1}{4}} \delta^{\frac{1}{2}} \mathrm{E}^{\frac{1}{4}}\right\},
$$

and so all quantities, $w_{\mathrm{B}}$ included, fall off exponentially away from the bottom of the slope. Substituting into (23), we obtain

$$
w_{\mathrm{B}}=\frac{-2^{\frac{1}{2}} \beta^{2} \delta}{\mathrm{E}^{\frac{1}{2}}} \cdot\left[A M^{\prime \prime}(\lambda)+B U^{\prime \prime}(\lambda)\right] \text { on } \xi=\delta .
$$

This is shown, for varying $\sigma$, in Fig. 8. Both $w_{r}$ and
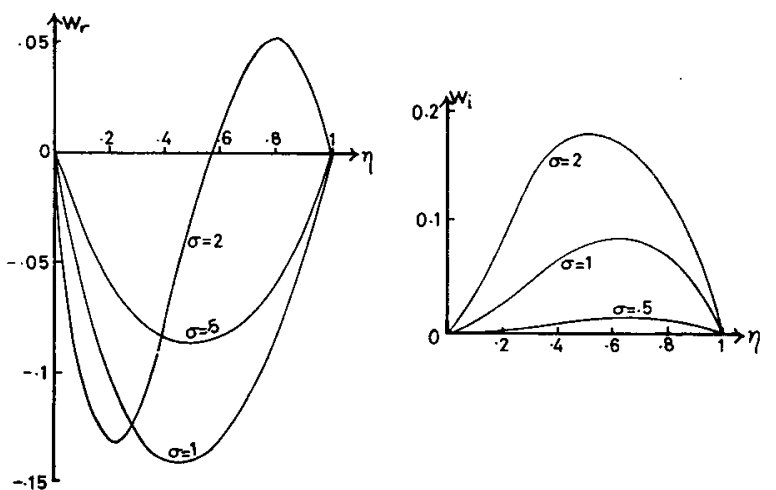

JiG. 7. Vertical velocity as a function of $\eta$ for various $\sigma$. $w_{\mathrm{B}}=\mathrm{E}^{1} \tau^{(y)}(1)\left[w_{r}+i w_{i}\right]$.

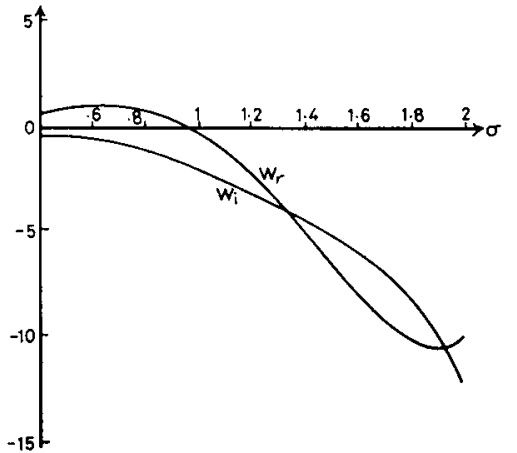

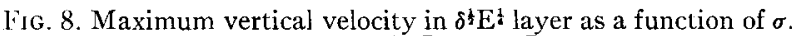
$w_{\mathrm{B}}=\mathrm{E}^{\frac{1}{*}} \tau^{(y)}(1)\left[w_{r}+i w_{i}\right]$.

$w_{i}$ are of order $4 \mathrm{E}^{i}$ or more for $\sigma>1.3$, and can have magnitudes as large as $10 \mathrm{E}^{\frac{1}{4}}$ for $\sigma \approx 2$; $w_{r}$ is negative for $\sigma>0.9, w_{i}$ is always negative. In terms of observations, this means that, for $\sigma>1$, there will be a vertical velocity near the bottom of the slope, probably large enough to be detectable, which has the same direction as the locally-produced up- or downwelling at the top of the slope. This phenomenon appears qualitatively similar to the persistent "areas of secondary upwelling" off the coast of West Africa observed by Jones and Folkard (1970), and by Hart and Currie (1960), and is produced by the shear between the slope and flat bottom regions.

One case of the circulation in the $(\eta, z)$ plane is shown in Fig. 9.

It is obviously desirable to investigate qualitatively the direct lateral friction layer. We have not solved for it in detail, but will attempt a qualitative description on the assumption that a well-behaved solution exists. The $E^{i}$ direct lateral friction layer exists to smooth out $u_{x}$ and $w\left(v, v_{x}\right.$ and $u$ are already continuous) between the slope and flat bottom regions. The vertical velocities are of order $\mathrm{E}^{\frac{1}{2}}$ and match onto those produced in the $\delta^{\frac{1}{2}} \mathrm{E}^{\frac{1}{2}}$ layer, and die away as $\xi=\delta$ is approached. It therefore seems plausible that the vertical velocity, as a function of $\xi$, is always of the same sign in the direct lateral friction layer, giving a localized area of strong upwelling. Even if $w_{\mathrm{B}}$ were to change sign in the $\mathrm{E}^{\frac{1}{2}}$ layer, it is unlikely to reach as large a magnitude as that in the $\delta^{\frac{1}{2}} E^{\frac{1}{4}}$ layer.

We have seen then that an important phenomenon in this solution is that wind-produced upwelling at the east coast also is associated with secondary weaker upwelling in a thin layer extending from the bottom of the slope to the surface. Because of the difficulty of measuring such small vertical currents directly, observations of up- or downwelling are made indirectly by means of contour diagrams of temperature, salinity, phosphates, etc. Vertical velocities of order $5 \mathrm{E}^{\frac{1}{2}}$ should show up fairly clearly on these.

It must finally be pointed out that the "gyre" structure implicit in the $e^{i \alpha y}$ dependence implies that 
secondary upwelling will occur even in areas in which there is no local wind, being forced by conditions a quarter of a wavelength away. This suggestion has been made by Yoshida (1967), considering a two-layer model, although he connected this with the direction of propagation of Kelvin waves, rather than a steady phenomenon.

\section{The west coast solution}

To complete the solution, begun in Section 3, we must now solve the problem in the vicinity of the western boundary. Once again, we find a Munk layer, thickness $\mathrm{O}\left(\mathrm{E}^{\frac{1}{3}}\right)$, over the flat bottom. In the Munk (1950) or Pedlosky (1968) theory, its function was to bring the northward velocity, amplified within this layer, to zero. Unlike the eastern boundary, this part of the solution is modified only quantitatively by the presence of the slope. Thus, over the flat bottom,

$$
\begin{aligned}
p=\frac{1}{\beta} \int_{1}^{x} w_{\mathbf{E}} d x+G+F_{1} \exp \left[\frac{\xi \beta^{\frac{1}{3}}}{2 \mathrm{E}^{\frac{1}{3}}}\left(3^{\frac{1}{2}} i-1\right)\right] \\
+F_{2} \exp \left[\frac{\xi \beta^{\frac{1}{3}}}{2 \mathrm{E}^{\frac{1}{3}}}\left(-3^{\frac{1}{3}} i-1\right)\right],
\end{aligned}
$$

where $\xi$ is now $(x-\delta) ; G$ was determined in Section 3; $F_{1}, F_{2}$ are unknown constants. Over the western slope, a similar equation to (10) is satisfied, with some sign changes:

$$
\frac{\mathrm{E}^{\frac{1}{2}}}{2^{\frac{1}{2}}} p_{x x}+\beta x p_{x}+i \alpha p=\delta w_{\mathbf{E}}(0),
$$

with solution

$$
p=\frac{-i \delta w_{\mathrm{E}}}{\alpha}+A_{1} \tilde{M}(x)+B_{1} \tilde{U}(x),
$$

where

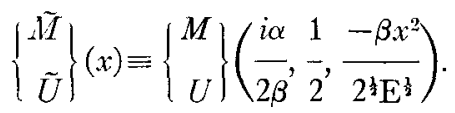

The lateral bottom frictional layer on $x=\delta$ contributes an extra term

$$
C \exp \left[\xi / 2 ! \delta^{\frac{1}{2}} \mathrm{E}^{i}\right],
$$

and, as before, the similar layer on $x=0$ is absorbed into the direct lateral friction layer. The boundary condition on the flux at $x=0$ yields

$$
B_{1}=\frac{\delta \mathrm{E}^{!} 2^{\frac{3}{3}} \beta^{\frac{1}{2}} \Gamma\left(\frac{i \alpha}{2 \beta}+1\right)}{\alpha \Gamma\left(\frac{1}{2}\right)} \tau^{(y)}(0), \mathrm{O}\left(\mathrm{E}^{\frac{1}{2}}\right) .
$$

The matching conditions (16a), (16b) gives $A_{1}, F_{1}, F_{2}$ and $C$ which are of order $\left(\mathrm{E}^{\frac{1}{2}}, \mathrm{E}^{\frac{1}{2}}, \mathrm{E}^{\frac{1}{2}}, \mathrm{E}^{7 / 12}\right)$ even if the

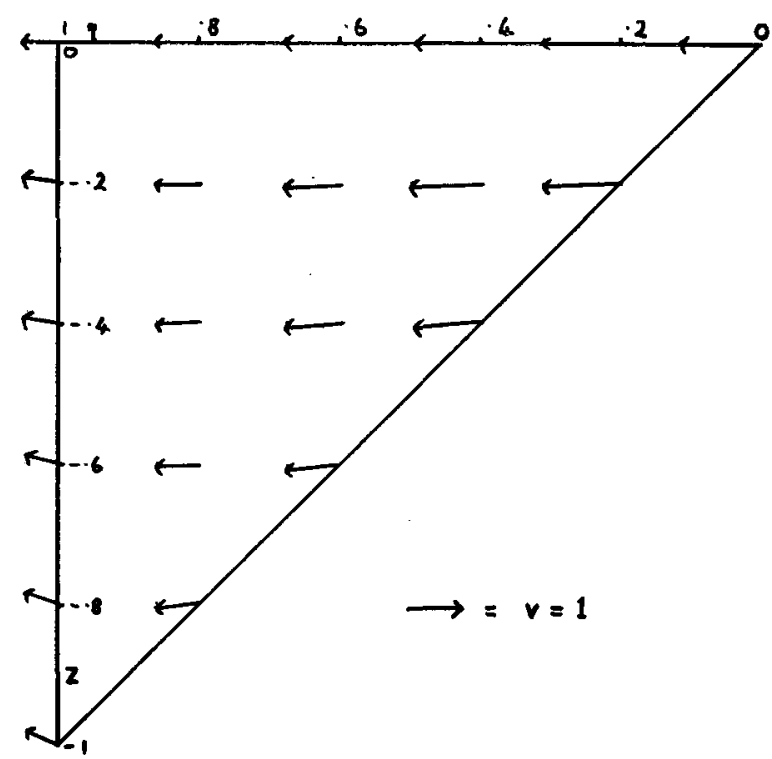

FIG. 9. Circulation at $y=0$ in the $(\eta, z)$ plane for $\sigma=\tau^{(y)}=1$ (i.e., downwelling at the coast). The velocity plotted is $\left(E^{-\frac{1}{3}} u, \mathrm{E}^{-\frac{1}{w}} w\right)$. Note the upwelling on $\eta=1$ due to the $\delta^{\frac{1}{2}} \mathrm{E}^{\mathfrak{t}}$ layer (weak for this value of $\sigma$ ). Superimposed on this should be the Ekman layer fluxes, shown qualitatively in Figs. 2 and 3.

local $\tau^{(y)}$ is zero. We find

$$
\left.\begin{array}{l}
A_{1}=\frac{1}{M-M^{\prime}}\left[\frac{-1}{\beta} \int_{1}^{0} w_{1} d x+G-\left(U-U^{\prime}\right) B\right] \\
F_{2}=\frac{3+3^{\frac{1}{2}} i}{6}\left[A_{1} M^{\prime}+B_{1} U^{\prime}\right] \\
F_{1}=\frac{1-3^{\frac{1}{2}} i}{2} F_{2}
\end{array}\right\},
$$

where $M, U$, etc., are evaluated with arguments $(i \alpha / 2 \beta$, $\left.\frac{1}{2},-\lambda\right)$. From this we may see that $v\left(=p_{x}\right)$ in the Munk layer is zero to $\mathrm{O}\left(\mathrm{E}^{1 / 6}\right)$ on $x=\delta$, unlike the corresponding case on the eastern boundary. Then, over the flat bottom, $v$ is $O\left(E^{1 / 6}\right)$ and is given by

$$
v=\left(\frac{\beta}{\mathrm{E}}\right)^{\frac{1}{3}} F_{2} i\left(i 3^{\frac{1}{2}}+1\right) \exp \left(-\frac{\xi \beta^{\frac{1}{3}}}{2 \mathrm{E}^{\frac{1}{3}}}\right) \sin \frac{3^{\frac{1}{2}} \xi \beta^{\frac{1}{3}}}{2 \mathrm{E}^{\frac{1}{3}}},
$$

which is similar to the vertical wall case; over the slope

$$
\begin{aligned}
v=\frac{-2^{\frac{1}{2}} \beta \delta}{\mathrm{E}^{\frac{1}{2}}}\left[A _ { 1 } M ^ { \prime } \left(\frac{i \alpha}{2 \beta}, \frac{1}{2},\right.\right. & \left.\frac{-\beta x^{2}}{2^{\frac{1}{2}} \mathrm{E}^{\frac{1}{2}}}\right) \\
& \left.+B_{1} U^{\prime}\left(\frac{i \alpha}{2 \beta}, \frac{1}{2}, \frac{-\beta x^{2}}{2^{\frac{1}{2}} \mathrm{E}^{\frac{1}{2}}}\right)\right] .
\end{aligned}
$$

Evaluation of the northward fluxes near the western boundary reveals that the effect of local upwelling has been to transfer a "parcel" of flux from the west coast 


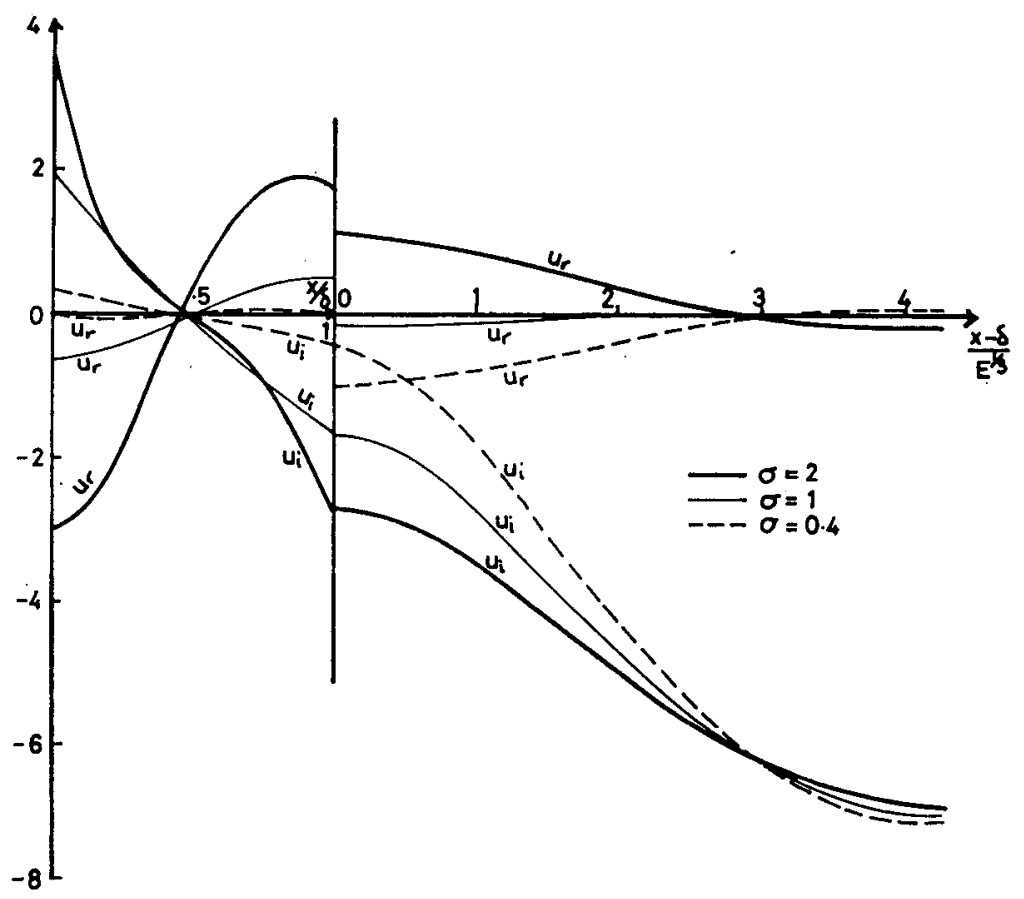

FIG. 10. The terms of $u$ proportional to $\tau^{(y)}(0)$ on the west coast as a function of $x$ :

$$
u=\mathrm{E}^{\frac{1}{2}} \tau^{(y)}(0)\left[u_{r}+i u_{i}\right] \text {. }
$$

to the east, of magnitude

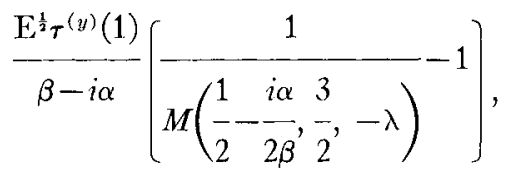

another form of (20).

We shall now describe how the velocities and fluxes are distributed between the slope and Munk layers, although not in as much detail as for the eastern side of the basin. This is for two reasons: 1) since the details of the velocity structure depend linearly on the three quantities

$$
\int_{0}^{1} \tau^{(x)} d x, \quad \tau^{(y)}(1), \quad \tau^{(y)}(0)
$$

it is difficult to give a complete picture of the flow without a large number of diagrams; and 2) nonlinear effects are likely to be important near the western

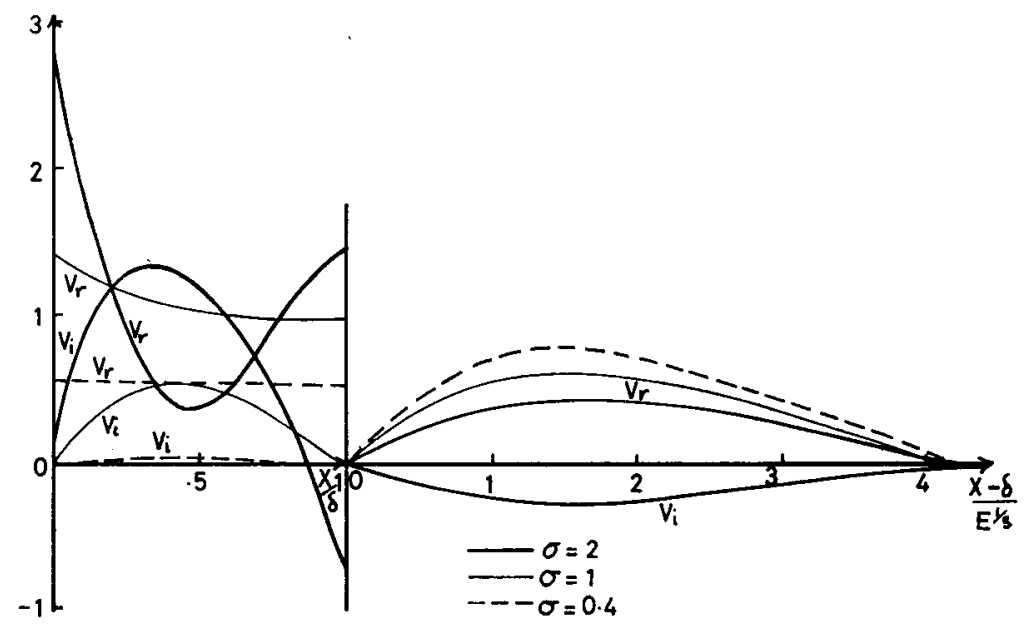

FIG. 11. The terms of $v$ proportional to $\tau^{(y)}(0)$ on the west coast as a function of $x$ :

$\begin{array}{ll}\text { For } x<\delta, & v=\mathrm{E}^{\frac{1}{}} \tau^{(y)}(0)\left[v_{r}+i v_{i}\right] \\ \text { For } x>\delta, & v=\mathrm{E}^{1 / 6} \tau^{(y)}(0)\left[v_{r}+i v_{i}\right]\end{array}$ 
boundary, which would invalidate the analysis of this section.

Instead, we present only the terms of the horizontal velocities proportional to $\tau^{(y)}(0)$ for $\sigma=0.4,1$, and 2 (Figs. 10 and 11). Note that the $x$ axis has been divided into the regions

$$
\text { (a) } 0<x / \delta<1, \quad \text { (b) } 0<(x-\delta) / \mathrm{E}^{\frac{1}{3}}<4 \text {, }
$$

and, in (b), the non-asymptotically vanishing terms have been included. The graphs of those terms of $u, v$ proportional to $\tau^{(y)}(1)$ and

$$
\int_{0}^{1} \tau^{(x)} d x
$$

are of a similar nature and are not shown here.

Fig. 10 shows that, for all $\sigma$, the terms of $u$ proportional to $\tau^{(y)}(0)$ change sign near $x / \delta=0.5$. So, in a similar fashion to the east slope, there is a point on the west slope for which $u=0$ [due to the local $\tau^{(y)}$ ]. Note that this does not apply to the other two terms which make up $u$. In general, as $\sigma$ increases, the magnitude of the horizontal velocities over the slope increase and decrease in the Munk layer.

We have noted that the northward fluxes in the slope and Munk layers are both of order $\mathrm{E}^{\frac{1}{2}}$. Fig. 12 shows the terms of the northward fluxes proportional to $\tau^{(y)}(0)$ [again the other terms are similar, and not shown], from which we see that the fluxes are in fact numerically of the same order also. For $\sigma=2$ there is almost as much northward flux over the slope as in the Munk layer; this may be part of the explanation of why the Gulf Stream follows the depth contours before separation.

In order to test the above results, Eqs. (1)-(4) were posed in terms of a streamfunction $\psi$ for the vertically integrated flow and solved numerically for varying width of continental slope. Contours of $\psi$ are given for two cases in Fig. 13. For comparison, the maximum value of $\psi$ for the case of a flat bottom was 3.51. From

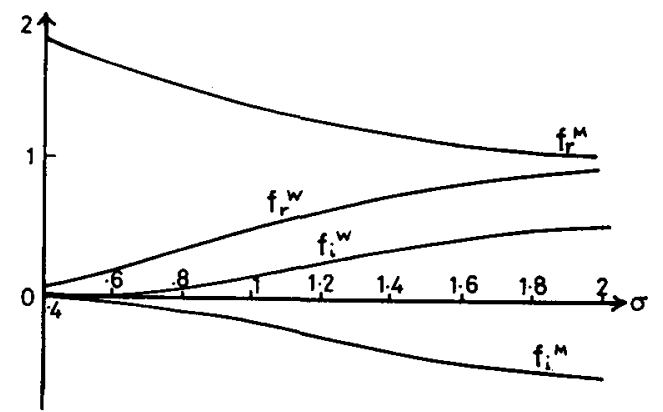

lig. 12. The terms of the northward flux at the west coast proportional to $\tau^{(y)}(0)$ :

Flux in slope layer $=\mathrm{E}^{\hat{b}} \tau^{(y)}(0)\left[f_{r}{ }^{w}+i f_{i}^{w}\right]$, Flux in Munk layer $=\mathrm{E}^{\frac{1}{2}} \tau^{(y)}(0)\left[f_{r^{M}}+i f_{i}{ }^{M}\right]$.
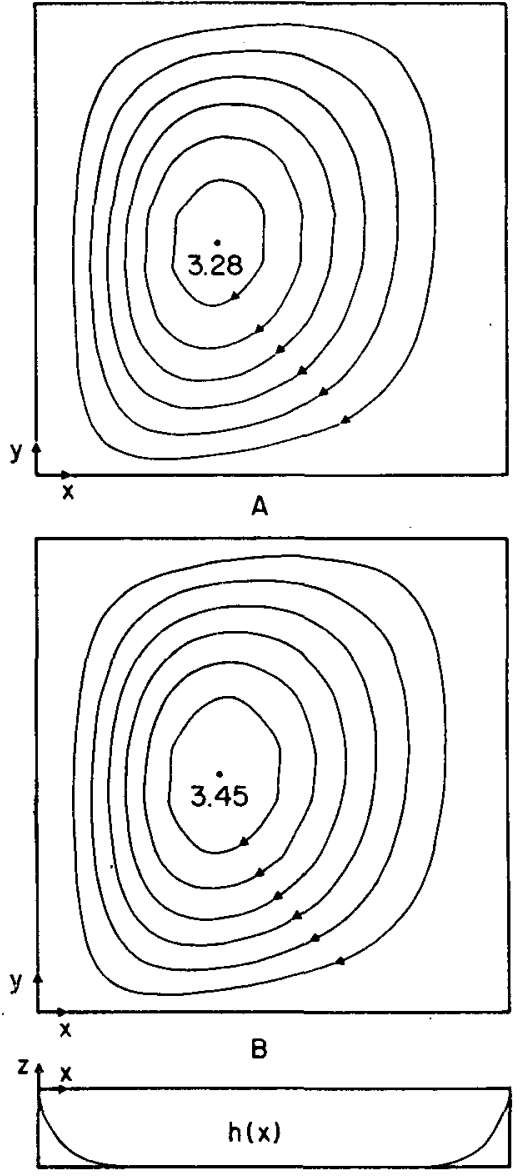

FIG. 13. Contours of streamfunction $\psi$ for integrated circulation (maximum value as indicated):

$$
\left.\tau^{(x)}=-1, h=1-\left(1-h_{0}\right)\{\exp (-x / \delta)+\exp [(x-1) / \delta)]\right\},
$$

where $h_{0}=0.1, \delta=0.05 ; \mathrm{E}=10^{-3}$. In case A, $\tau^{(y)}=0$, and case $\mathrm{B}$, $\tau^{(y)}=i$

this we see again the following effects:

1. The maximum flux is reduced by the continental slope, confirming the results of Schulman and Niiler (1970).

2. The east coast flux is at most $3 \%$ of the main west boundary flux, i.e., the effect of sloping boundaries on the global circulation is small.

3. Since frictional effects dominate on the slope, there is little tendency for southward deflection of streamlines (i.e., conservation of potential vorticity) unless the slope is wider than $O\left(E^{\frac{1}{3}}\right)$, when the Schulman and Niiler analysis applies.

\section{Conclusions}

We have seen that the dynamics of large-scale, homogeneous ocean circulation for a basin bounded by sloping, but not vertical, walls, differs significantly from the more usual vertical-wall theory in the following 
respects:

1) The motion on the slope is geostrophic, despite the small length scales involved.

2) The existence of bottom flux forces strong longshore currents at eastern boundaries as well as western, although these currents are smaller in magnitude than those (in linear theories) in western boundary currents. This explanation for eastern boundary currents is different from the Sverdrup et al. (1942) suggestion of baroclinically-driven currents.

3) This effect can only produce northward fluxes on the eastern boundary of order 1 sverdrup.

4) An area of secondary upwelling, sited near the bottom of the continental slope, is predicted, agreeing qualitatively with observations, given our choice of $\nu_{I I}$ and $\nu_{V}$. This upwelling will even occur in the absence of local winds. We have considered here only a discontinuous change in bottom slope. In the more physical case of a smoother topography, $h_{x}$ changes from its slope value to zero in a horizontal distance $\theta$, say. The differing dynamics over the slope and flat bottom regions results in a change of magnitude of the horizontal velocities which takes place over a horizontal distance which is also of order $\theta$. The resulting horizontal velocity shear will again force secondary upwelling, but in general this will be weaker and more diffuse than that considered here.

5) A good proportion of the (linear) western boundary current exists on the slope; the largest northward velocities "hug" the foot of the stope.

It may also be noted that there is little tendency for deflection of streamlines on the slope in the sense that would be associated with potential vorticity conservation.

The precise formulation of the model, and its quantitative results, depend on the hypothesis of constant eddy coefficients of viscosity. It is likely that the dynamics of the turbulent bottom friction layer (for example) will be quite different from that considered here. However, the existence of a flux within this layer must be associated with a bottom stress, which is in turn associated with--presumably-northward velocities in the interior. There seems little reason to suspect that the qualitative behavior of the other frictional layers will differ in essential detail from the description given here. It is not obvious that large-scale upwelling could take place in the presence of stratification. The returning surface flux may have to be distributed somewhat faster to the interior than in the manner presented here, depending on the magnitude of the turbulent heat diffusion near the boundaries. This problem remains for further study.

Acknowledgments. The author would like to thank Dr. A. E. Gill for suggesting the topic, and for much advice during the work which was carried out at the Department of Applied Mathematics and Theoretical Physics of the University of Cambridge, England, under a grant from the Science Research Council of Great Britain.

\section{APPENDLX}

\section{The Bottom Ekman layer}

Consider the vicinity of a slope $z=z(x)$. Let the derivative $d z / d x$ be $-s$, say. Then the equation satisfied near the slope are

$$
\begin{aligned}
& f v=-\left(\nu_{I I} u_{x x}+\nu_{V} u_{z z}\right), \\
& f u=+\left(\nu_{H} v_{x x}+\nu_{V} v_{z z}\right)
\end{aligned}
$$

since the pressure is hydrostatic, where the velocity $=\left(u^{I}, v^{I}, w^{I}\right)+(u, v, 0)$, with $\left(u^{I}, v^{I}, w^{I}\right)$ being the velocity away from the slope.

Putting $\tilde{\omega}=u+i v$, the solution of (A1), (A2) satisfying

is

$$
\begin{gathered}
\tilde{\omega} \rightarrow 0, \quad \text { as } \quad z-z(x) \rightarrow \infty, \\
\tilde{\omega}=-\tilde{\omega}^{I}=-\left(u^{I}+i v^{I}\right), \text { on } z=z(x),
\end{gathered}
$$

where

$$
\widetilde{\omega}=-\tilde{\omega}^{I} \exp [-\lambda(z+s x)]
$$

$$
\lambda=\frac{1+i}{2^{\frac{1}{2}}}\left(\frac{f}{\nu_{H} s^{2}+\nu_{V}}\right)^{\frac{1}{2}}
$$

providing

$$
\left|\frac{d s}{d x}\right| \ll\left(\frac{\nu_{H}}{f}\right)^{\frac{1}{2}}
$$

i.e., $s$ is locally constant. Whence

or

$$
\int_{z=z(x)}^{z+s x=\infty} \tilde{\omega} d z=\frac{-\tilde{\omega}^{I}}{\lambda}
$$

$$
\begin{aligned}
& \int_{z=z(x)}^{\infty} u d z=-\left(u^{I}+v^{I}\right)\left(\frac{\nu_{H} s^{2}+\nu_{V}}{2 f}\right)^{\frac{1}{2}}, \\
& \int_{z=z(x)}^{\infty} v d z=\left(u^{I}-v^{I}\right)\left(\frac{\nu_{I I} s^{2}+\nu_{V}}{2 f}\right)^{\frac{1}{2}} .
\end{aligned}
$$

Continuity then implies that $w_{B}$, the vertical velocity above the Ekman layer, is

$$
\begin{aligned}
w_{B}=-u^{I} h_{x}+\frac{\partial}{\partial x}\left[\left(u^{I}+v^{I}\right)\right. & \left.\left(\frac{\nu_{H} s^{2}+\nu_{V}}{2 f}\right)^{\frac{1}{2}}\right] \\
& +\frac{\partial}{\partial y}\left[\left(u^{I}-v^{I}\right)\left(\frac{\nu_{H} s^{2}+\nu_{V}}{2 f}\right)^{\frac{1}{2}}\right] .
\end{aligned}
$$

If $s$ changes only slowly with $x$, this may be replaced by

$w_{\mathrm{B}}=-u^{I} h_{x}+\left(\frac{\nu_{H} s^{2}+\nu_{V}}{2 f}\right)^{\frac{1}{2}}\left[\left(u_{x}^{I}+v_{y}{ }^{I}\right)+\left(v_{x}{ }^{I}-u_{y} I\right)\right]$

This result is valid providing the $y$ derivatives of $u$ and 
$v$ are negligible; this can only break down very near the equator, where the thickness of the Ekman layer increases. The condition for validity is

$$
\frac{\nu_{H} \beta^{2}}{f^{3}} \ll 1
$$

which is satisfied, even for $\nu_{I I}=10^{8} \mathrm{~cm}^{2} \mathrm{sec}^{-1}$, for latitudes $\gtrsim 2^{\circ}$.

Nondimensionally, with $\mathrm{E}_{H}=\mathrm{E}_{V}=\mathrm{F}, \quad(\mathrm{A} 3)-(\mathrm{A} 5)$ become

$$
\begin{aligned}
& \int u d z=\frac{-\mathrm{E}^{\frac{1}{2}}}{2^{\frac{1}{2}} \cos K}(u+v), \\
& \int v d z=\frac{-\mathbf{E}^{\frac{1}{2}}}{2^{\frac{1}{2}} \cos K}(v-u),
\end{aligned}
$$

and, assuming that, where $w_{B}$ is important,

$$
\begin{gathered}
\underset{\partial x}{\stackrel{\partial}{\partial}} \frac{\partial}{\partial y}, \quad|v| \gg|u|, \\
w_{\mathrm{B}}=-u h_{x}+\frac{\mathrm{E}^{\frac{1}{2}}}{2^{\frac{1}{2}} \cos K} v_{x} .
\end{gathered}
$$

Note that $\left(A 5^{\prime}\right)$ is not the same result as that obtained by Johnson $e$ t al. (1971) since they assumed $x$ derivatives to be $\mathrm{O}(1)$ within the bottom Ekman layer.

\section{REFERENCES}

Abramowitz M., and I. A. Stegun, 1965: Handbook of Mathematical Functions. New York, Dover, 1046 pp.

Bryan, K., 1963: A numerical investigation of a non-linear model of a wind-driven ocean. J. Atmos. Sci., 20, 594-606.
Clarke, R. A., and N. P. Fofonoff, 1969: Oceanic flow over varying bottom topography. J. Marine Res., 27, 226-240.

Ellett, D. J., 1968: The cold 'winter-water' of Rockall Bank. ICES CM 1962 Hydrography Committee Doc. C:24.

Hart, T. J., and R. I. Currie, 1960: The Benguela Current. Discovery Rept., No. 31.

Holland, W., 1967: On the wind-driven circulation in an ocean with bottom topography. Telhis, 19, 582-600.

Janowitz, G. S., 1970: The coastal boundary layers of a lake. Tellus, 22, 585-596.

Johnson, J. A., 1968: A three-dimensional model of the winddriven ocean circulation, J. Flivd Mech., 34, 721-734.

, C. B. Fandry and C. M. Leslie, 1971: On the variation of ocean circulation produced by bottom topography. Tellus, 23, $113-121$.

Jones, P. G. W., and A. R. Folkard, 1970: Chemical oceanographic observations off the coast of northwest Africa, with special reference to the process of upwelling. Conseil Intern. l'Exploration mer. Extrait Rapp. Proc. Verb., 159, 38-60.

Munk, W. H., 1950: On the wind-driven circulation. J. Meteor., $7,79-93$.

Pedlosky, J., 1968: An overlooked aspect of the wind-driven ocean circulation. J. Fluid Mech., 32, 809-821.

---, 1969: The linear theory of the circulation of a stratified ocean. J. Fluid Mech., 35, 185-205.

Rhines, P., 1970: Edge-, bottom-, and Rossby waves in a rotating stratified fluid. Geophys. Fluid Dyn., 1, 273-302.

Schulman, E. E., and P. P. Niiler, 1970: Topographic effects on the wind-driven ocean circulation. Geophys. Fhid Dyn., 1, $439-462$.

Stommel, H., 1948: The western intensification of wind-driven ocean currents. Trans. Amer. Geophys. Union, 29, 202-206.

---, 1955: The Gulf Stream. Berkeley, University of California Press, $202 \mathrm{pp}$.

Sverdrup, H. U., 1947: Wind-driven currents in a baroclinic ocean. Proc. Natl. Acad. Sci., 33, No. 11, 318-326.

—- M. W. Johnson and R. H. Fleming, 1942: The Oceans. New York, Prentice-Hail, $1087 \mathrm{pp}$.

Tomczak, M., 1970: Eine lineare Theorie des stationearen Auftriebs in stetig geschichteten Meer. Deut. Hydrogr. Z., 23, 214-234.

Yoshida, K., 1967: Circulation in the eastern tropical oceans with special references to upwelling and undercurrents. Japan $J$. Geophys., 4, 1-75. 